\title{
Measuring Heart Rate Variability by means of Information Entropies based on Choi-Williams Distribution
}

\author{
Montserrat Vallverdú, Francesc Clariá, Umberto Melia, Antonio Bayés de Luna, Pere Caminal
}

\begin{abstract}
The Shannon entropy theory was applied to the Choi-Williams time-frequency distribution (CWD) of cardiac time series (RR series) in order to extract entropy information in both time and frequency domains. From this distribution, four indexes were defined: (1) instantaneous partial entropy; (2) spectral partial entropy; (3) instantaneous complete entropy; (4) spectral complete entropy. These indexes were used for analyzing the heart rate variability of ischemic cardiomyopathy patients (ICM) with different sudden cardiac death risk. The results have shown that the values of these indexes tend to decrease, with different proportion, when the severity of pathological condition increases. Statistical differences ( $p$-value $<0.0005)$ of these indexes were found comparing low risk and high risk of cardiac death during night and between daytime and nighttime periods of ICM patients. Finally, these indexes have demonstrated to be useful tools to quantify the different complex components of the cardiac time series.
\end{abstract}

\section{INTRODUCTION}

Cardiovascular diseases represent the most common cause of death worldwide [1] and their high incidence has motivated the development of quantitative markers, in order to identify the presence of cardiac pathologies and the risk of suffering cardiac death.

Several studies used heart rate variability (HRV) analysis as a non-invasive method for diagnosis of a large number of cardiovascular diseases [2], which may involve changes in the autonomic nervous system modulation and tone, thus facilitating the diagnosis and prognosis of cardiopathies and neuropathies. Unfortunately, the stratification of risk groups in patients with heart failure is an issue that has yet to be resolved.

Methods for quantifying HRV by means of the RR series (time intervals between consecutive heart beats) are based on time domain, frequency domain, geometric and nonlinear techniques [2-5]. However, given the intrinsic nonlinear nature in time and frequency of the cardiac regulatory mechanisms, a more insightful description in nonlinear timefrequency representation could help to describe the dynamical changes of HRV.

M. Vallverdú, U.S.P. Melia and P. Caminal are with Dept. ESAII, Centre for Biomedical Engineering Research, CIBER, Universitat Politècnica de Catalunya, Barcelona, Spain; email: \{montserrat.vallverdu,umberto.melia, pere.caminal\}@upc.edu

F. Clarià is with Dept. IIE, Lleida University, Spain; email: claria@diei.udl.es.

A. Bayés de Luna is with the Catalan Institute of Cardiovascular Sciences, Barcelona, Spain; email: abayes@csic-iccc.org
The classical Shannon entropy measures the average information provided by a set of events and proves its uncertainty. This measure is shown as a natural candidate for quantifying the complexity of physiological signals. Also the level of chaoticity may be measured using entropy. Higher entropy represents higher uncertainty and a more irregular behavior of a signal. Entropy can even explain how linked complex systems interact and exchange information. The quantification of the magnitude of this information becomes a goal in the study of physiological signals.

The time-frequency representation (TFR) technique generalizes the concept of the time and frequency domains to a joint time-frequency function that indicates how the frequency content of a signal changes over time [6,7]. Complexity studies based on entropy functional take advantage of the analogy between signal energy densities and probability densities [8]. While the instantaneous and spectral amplitudes behave as one-dimensional densities of signal energy in time and in frequency, TFR tries to act as two-dimensional energy densities in both time and frequency [9]. Based on these concepts, the TFR information estimated by a probability density function of a signal either in time or in frequency domain permits to define new indexes to quantify the complexity content of a signal.

Four indexes [10] were defined by calculating entropy of the Choi-Williams distribution (CWD) [6] with respect to time or frequency, by using the probability mass function at each time instant taken independently or by using the probability mass function of the entire CWD.

\section{MATERIALS AND METHODS}

\section{A. Databases and Preprocessing}

\section{Synthetic Signals}

In order to study the performances of the proposed indexes, 200 synthetic signals were designed. A $S_{\text {signal }}$ process, used in previous studies [11,12], was defined as $S_{\text {signal }}=(1-z) x+z y$, where $z$ is a random variable that is equal to 1 with probability $p$ and equal to 0 with probability $1-p$. The parameter $p$ varied linearly from 0.9 to 0.1 . The variable $x$ is a periodic sequence with a frequency component of $0.0975 \mathrm{~Hz}$, and $y$ the $H x$ obtained from Henon map [13] with chaotic behavior (1), using the canonic values $\mathrm{a}=1.4$ and $\mathrm{b}=0.3$, and taking $H x(0)=0.5$ and $H y(0)$ $=0.5$ as initial conditions.

$$
\left\{\begin{array}{l}
H x(n+1)=1-a H x^{2}(n)+H y(n) \\
H y(n+1)=b H x(n)
\end{array}\right.
$$

Hence, these synthetic signals evolved from chaos to 
periodicity. The synthetic signals had a length of 30000 samples with a sampling frequency of $1 \mathrm{~Hz}$.

\section{HRV Series}

Patients with Heart Failure were analyzed in the present work. All these patients had symptomatic chronic heart failure (NYHA class II-III) and were treated according to institutional guidelines. The investigation was conforming to the recommendations of the Declaration of Helsinki, the study was approved by the Ethical Committee of the institution and all subjects gave their written informed consent before participation. A total of 150 patients with ischemic cardiomyopathy (ICM) at risk for cardiac death were enrolled in the present work. The inclusion criteria were: sinus rhythm, symptomatic chronic heart failure with NYHA functional class II or III, and ischemic etiology of heart failure.

After a follow-up of three years the following analysis was due: 138 survivor patients (SV) as a low risk (LR) group and 12 patients that suffered sudden cardiac death (SCD) as a high risk (HR) group. SCD patients were aged-matched with SV group, with $64.1 \pm 1.13$ year old and $91.8 \%$ male.

The RR series, intervals between consecutive beats, were obtained from 24h ECG-Holter recordings with a sampling frequency of $200 \mathrm{~Hz}$. Equidistant time series were obtained by linearly interpolating the RR interval series at a resampling rate of $1 \mathrm{~Hz}$. Furthermore, an adaptive and the ASEF filter [14] were applied to the RR series (figure 1a) in order to reduce the artifacts while frequency content is preserved (figure $1 \mathrm{~b}$ ). The $24 \mathrm{~h}$ RR series were analyzed and also in two periods, 10 hours during daytime (10h-20h) and 10 hours during nighttime (20h-6h).
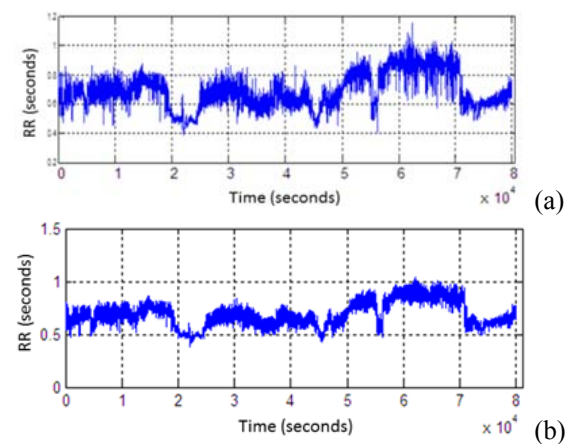

Figure 1. (a) Non-filtered and (b) filtered RR series by using ASEF

\section{B. Time-Frequency Representation}

Choi-Williams distribution $C W D(t, f)[6,7]$ is obtained by convoluting the Wigner distribution (2)

$$
W D x(t, f)=\int_{-\infty}^{\infty} x(t+\tau / 2) x^{*}(t-\tau / 2) e^{-j 2 \pi f \tau} d \tau
$$

and the $C W$ exponential (3)

$$
C W(t, f)=\sqrt{\frac{4 \pi}{\sigma_{c}}} e^{-4 \pi^{2} \frac{(t f)^{2}}{\sigma_{c}}}
$$

where parameter $\sigma_{c}$ [15] permits $C W D$ to reduce the crossterms and to preserve marginal properties and instantaneous frequency.

The spectral power is defined as

$$
\operatorname{SpPow}(f)=\int_{-\infty}^{\infty} C W D(t, f) d t
$$

Finally, the $C W D(t, f)$ was normalized by the total power calculated as the area under $\operatorname{SpPow}(f)$.

Furthermore, the classical spectral power entropy $(E n C L s p)$ is defined as

$$
\operatorname{EnCLsp}(t)=-\int_{f 1}^{f 2} C W D(t, f) \log _{2}(C W D(t, f)) d f
$$

\section{Instantaneous Entropy and Spectral Information Entropy}

The probability mass function (PMF) was defined for a time instant $t_{k}$ with respect to frequency as $p T_{P M F}\left(t_{k}, i\right)=$ $P_{C W D}\left(C W D\left(t, f_{i}\right) \mid t=t_{k}\right)$ and for frequency value $f_{k}$ with respect to time as $p F_{P M F}\left(i, f_{k}\right)=P_{C W D}\left(C W D\left(t_{i}, f\right) \mid f=f_{k}\right)$, after the quantization of the $C W D\left(t_{k}, f\right)$ and $C W D\left(t, f_{k}\right)$, respectively, in $n=32$ equidistant levels.

The two distributions, quantization-time $p T_{P M F}(t, i)$ and quantization-frequency $p F_{P M F}(i, f)$, were obtained for each time instant and frequency value. In this way, the two distributions represent partial $(p)$ distribution of PMF with respect to time or to frequency, since in each time instant $\left(t_{k}\right)$ and frequency value $(f k)$ the PMF is only related to that time instant $\left(t_{k}\right)$ or frequency value $\left(f_{k}\right)$. In a similar way, the complete (c) PMF distribution quantization-time and quantization-frequency were calculated as $c T_{P M F}(t, i)=$ $P_{C W D}\left(C W D\left(t, f_{i}\right) \mid \forall t\right) \quad$ and $\quad c F_{P M F}(i, f) \quad=$ $P_{C W D}\left(C W D\left(t_{i}, f\right) \mid \forall f\right)$, respectively, after the quantization of the $C W D(t, f)$ in $n=32$ equidistant levels.

From this proposed methodology, four indexes were defined [10]:

- Instantaneous partial entropy

$$
\operatorname{EnPAins}(t)=-\sum_{\mathrm{i}=1}^{\mathrm{n}} p T_{P M F}(t, i) \log _{2}\left(p T_{P M F}(t, i)\right)
$$

- Spectral partial entropy

$$
\operatorname{EnPAsp}(f)=-\sum_{i=1}^{n} p F_{P M F}(i, f) \log _{2}\left(p F_{P M F}(i, f)\right)
$$

- Instantaneous complete entropy

$$
\operatorname{EnCOins}(t)=-\sum_{i=1}^{n} c T_{P M F}(t, i) \log _{2}\left(c T_{P M F}(t, i)\right)
$$

- Spectral complete entropy

$$
\operatorname{EnCOsp}(f)=-\sum_{i=1}^{n} c F_{P M F}(i, f) \log _{2}\left(c F_{P M F}(i, f)\right)
$$

All measures were calculated in the following frequency bands: Ultra-low frequency (ULF: $<0.003 \mathrm{~Hz}$ ), very low frequency (VLF: 0.003-0.04 Hz), low frequency (LF: 0.04- 
$0.15 \mathrm{~Hz}$ ), high frequency (HF: $0.15-0.4 \mathrm{~Hz}$ ) and total band frequency band (Tot).

Mean ( $m$ ) of EnPAins, EnPAsp, EnCOins, EnCOsp and $E n C L s p$, respectively, were calculated from the analyzed signals.

\section{Statistical Analysis}

A non-parametric test, the Mann-Whitney U test, was applied considering two groups: LR versus HR groups. Wilcoxon test was applied to analyze the statistical differences between daytime and nighttime periods. A significance level $\mathrm{p}$-value $<0.05$ was considered as significant. A discriminant linear function was built for every individual index in order to classify the subjects. The sensitivity (Sen), the specificity (Spe) and the area under the curve (AUC) were taken into account in this statistical analysis using leave-one-out cross-validation.

\section{RESULTS}

Figure 2 shows the results obtained applying the proposed methodology to a synthetic signal $\left(S_{\text {signal }}\right)$. Classical spectral power entropy (EnCLsp) (Figure 2a) does not show significant changes when the signal passes from a chaotic to a periodic behavior. On the contrary, EnPAins and EnCOins decrease from chaos to periodicity. During chaotic behavior EnPAins is higher than EnCOins, and then the two measures approach each other and converge in the zone with more periodicity. In Figure 2b, EnPAsp and EnCOsp show the location of the frequency component as the classical spectral power does. While EnCOsp maintains stable values for the remaining frequencies, EnPAsp presents irregular oscillations in the entire spectrum. Values of EnPAsp are higher than EnCOsp except for the frequency component of the signal $\mathrm{f}=0.0975 \mathrm{~Hz}$.

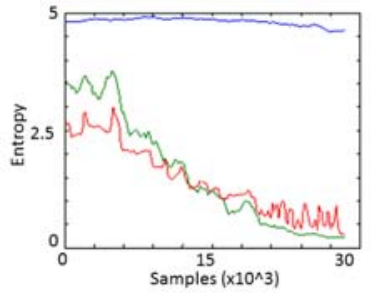

(a)

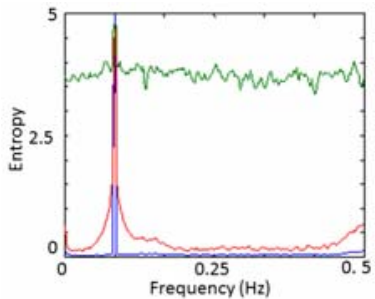

(b)
Figure 2. Synthetic Signal: (a) instantaneous complete entropy (EnCOins, red), instantaneous partial entropy (EnPAins, green) and classical spectral power entropy (EnCLsp, blue); (b) spectral complete entropy (EnCOsp, red), spectral partial entropy (EnPAsp, green) and classical spectral power (blue).

Table I contains the results of the mean values (mean \pm std) of those proposed indexes (EnPAins, EnPAsp, EnCOins and EnCOsp) with the best statistical classification power, calculated on each RR series of patients from $\mathrm{HR}$ and LR groups, and considering daytime, nighttime and $24 \mathrm{~h}$ periods. The indexes EnCOins(Tot), $\operatorname{EnCOsp}(L F)$ and $\operatorname{EnCOsp}(H F)$ presented the highest statistically significant differences during nighttime period when LR and HR groups were compared. This behavior was also observed during $24 \mathrm{~h}$ periods. The index $\operatorname{EnCOsp}(L F)$ permitted to classify the patients with a $S e n=60.1 \%, S p e=83.3 \%$ and $A U C=0.781$. The analysis performed during daytime period presented smallest statistical differences $(p$-value $<0.05)$ in those indexes, compared with nighttime and $24 \mathrm{~h}$ periods (pvalue $\leq 0.005)$. Only $\operatorname{EnCOins}(T o t)$ and $\operatorname{EnCOsp}(L F)$ could differentiate between LR and HR groups during daytime period. As observed in Table I, all these proposed indexes present higher values during LR group than HR group, indicating a highest complexity in time and frequency of the RR series in the LR group.

TABLE I

STATISTICAL MEASURES FOR THE INSTANTANEOUS ENTROPY AND SPECTRAL ENTROPY ANALYSIS: (HR) HIGH RISK AND (LR) LOW RISK OF SUFFERING SUDDEN CARDIAC DEATH

\begin{tabular}{|llll|}
\hline $\begin{array}{l}\text { Indexes } \\
\text { Mean }(\boldsymbol{m})\end{array}$ & $\begin{array}{l}\text { LR } \\
\text { mean } \pm \text { std }\end{array}$ & $\begin{array}{l}\text { HR } \\
\text { mean } \pm \text { std }\end{array}$ & $\begin{array}{l}\text { p- } \\
\text { value }\end{array}$ \\
\hline Daytime period & & & \\
\hline EnCOins $($ Tot $)$ & $0.435 \pm 0.0271$ & $0.252 \pm 0.0673$ & 0.049 \\
EnCOsp $(L F)$ & $0.397 \pm 0.0305$ & $0.202 \pm 0.0810$ & 0.041 \\
EnCOsp $(H F)$ & $0.233 \pm 0.0256$ & $0.0849 \pm 0.0367$ & n.s. \\
EnCOsp $(V L F)$ & $2.08 \pm 0.067$ & $1.65 \pm 0.321$ & n.s. \\
\hline Nighttime period & & & \\
\hline EnCOins (Tot) & $0.593 \pm 0.0264$ & $0.327 \pm 0.0466$ & 0.004 \\
EnCOsp(LF) & $0.595 \pm 0.0318$ & $0.245 \pm 0.0463$ & 0.001 \\
EnCOsp(HF) & $0.303 \pm 0.026$ & $0.099 \pm 0.0239$ & 0.005 \\
EnCOsp $(V L F)$ & $2.84 \pm 0.0606$ & $2.35 \pm 0.314$ & n.s. \\
\hline 24h period & & & \\
\hline EnCOins $($ Tot $)$ & $0.479 \pm 0.0132$ & $0.348 \pm 0.0397$ & 0.008 \\
EnCOsp $(L F)$ & $0.466 \pm 0.0156$ & $0.296 \pm 0.0505$ & 0.002 \\
EnCOsp $(H F)$ & $0.234 \pm 0.0119$ & $0.132 \pm 0.0277$ & 0.008 \\
EnCOsp $($ VLF) & $2.41 \pm 0.0399$ & $2.16 \pm 0.176$ & n.s. \\
\hline
\end{tabular}

TABLE II

Statistical Measures For the Classical SPECTRAL POWER ENTROPY ANALYSIS: HR, HIGH RISK AND LR LOW RISK OF SUFFERING SUDDEN

\begin{tabular}{|llll|}
\hline \multicolumn{5}{|c|}{ CARDIAC DEATH } \\
Mean $(\boldsymbol{m})$ & $\begin{array}{l}\text { LR } \\
\text { mean } \pm \text { std }\end{array}$ & $\begin{array}{l}\text { HR } \\
\text { mean } \pm \text { std }\end{array}$ & $\begin{array}{l}\text { p- } \\
\text { value }\end{array}$ \\
\hline Daytime period & & & \\
\hline EnCLsp $(H F)$ & $1.40 \pm 0.0307$ & $1.33 \pm 0.103$ & n.s. \\
EnCLsp $(L F)$ & $1.42 \pm 0.0299$ & $1.34 \pm 0.100$ & n.s. \\
EnCLsp $(V L F)$ & $1.60 \pm 0.0295$ & $1.48 \pm 0.1110$ & n.s. \\
\hline Nighttime period & & & \\
\hline EnCLsp $(H F)$ & $1.83 \pm 0.0336$ & $1.54 \pm 0.163$ & 0.020 \\
EnCLsp $(L F)$ & $1.85 \pm 0.0335$ & $1.55 \pm 0.163$ & 0.021 \\
EnCLsp $(V L F)$ & $2.06 \pm 0.0337$ & $1.73 \pm 0.178$ & 0.019 \\
\hline 24h period & & & \\
\hline EnCLsp(HF) & $1.58 \pm 0.0189$ & $1.44 \pm 0.0793$ & n.s. \\
EnCLsp $(L F)$ & $1.60 \pm 0.0188$ & $1.45 \pm 0.0789$ & 0.049 \\
EnCLsp $(V L F)$ & $1.79 \pm 0.019$ & $1.63 \pm 0.0837$ & n.s. \\
\hline
\end{tabular}

The results of the classical spectral power entropy $(E n C L s p)$ are presented in Table II. It can be observed that these indexes also present higher values during LR group than HR group, indicating a highest complexity in frequency of the RR series in the LR group. However, it is during nighttime that the main statistical differences are presented (p-value $<0.05$ ). Finally, comparing the statistical results from Tables I and II, an increased advantage of using the proposed indexes EnPAins, EnPAsp, EnCOins and EnCOsp can be observed. 
The results obtained for both types of indexes, the new proposed indexes and the classical spectral power entropy indexes, when daytime and nighttime periods were compared are presented in Table III. In this case, the ultralow frequency band in EnPAins and EnCOsp indexes could differentiate between daytime and nighttime periods (pvalue $<0.0005)$. Figures $3 a-3 b$ show the Choi-Williams distribution $C W D(t, f)$ of the RR series corresponding to this ultra-low frequency (ULF) band of a patient belonging to the survivor group (LR), from the daytime and nighttime periods, respectively. Lower values of the proposed new indexes (EnPAins (Tot), EnPAins $(U L F), \quad \operatorname{EnCOsp}(V L F)$, $\operatorname{EnCOsp}(U L F))$ and the classical spectral power entropy indexes $(\operatorname{EnCLsp}(H F), \quad \operatorname{EnCLsp}(L F), \quad \operatorname{EnCLsp}(V L F))$ are observed during daytime compared with nighttime periods, p-value $<0.0005$.

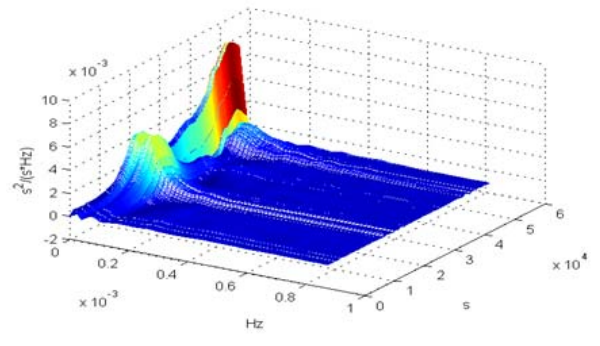

(a)

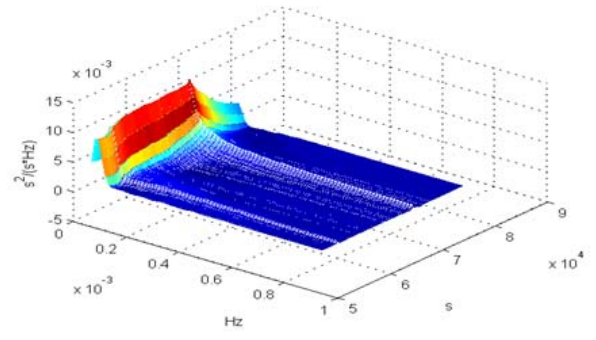

(b)

Figure 3. Choi-Williams distribution of the RR series for the ULF band: (a) daytime period and (b) nighttime period

TABLE III

Statistical MEASURES FROM THE ANALYSIS OF THE PROPOSED INDEXES COMPARING HRV PERIODS IN ISCHEMIC CARDIOMYOPATHY PATIENTS

\begin{tabular}{|c|c|c|}
\hline Periods & Daytime & Nighttime \\
\hline $\begin{array}{l}\text { Indexes } \\
\operatorname{Mean}(\mathrm{m})\end{array}$ & mean \pm std & mean \pm std \\
\hline EnPAins (Tot) & $0.435 \pm 0.0147$ & $0.639 \pm 0.0164^{*}$ \\
\hline EnPAins (ULF) & $1.616 \pm 0.0295$ & $1.982 \pm 0.0302 *$ \\
\hline EnCOsp $(V L F)$ & $2.05 \pm 0.0672$ & $2.80 \pm 0.0617^{*}$ \\
\hline $\operatorname{EnCOsp}(U L F)$ & $1.12 \pm 0.0319$ & $1.24 \pm 0.0307^{*}$ \\
\hline $\operatorname{EnCLsp}(H F)$ & $1.39 \pm 0.0294$ & $1.81 \pm 0.0339^{*}$ \\
\hline $\operatorname{EnCLsp}(L F)$ & $1.41 \pm 0.0286$ & $1.82 \pm 0.0339 *$ \\
\hline $\operatorname{EnCLsp}(V L F)$ & $1.59 \pm 0.0286$ & $2.03 \pm 0.0346^{*}$ \\
\hline
\end{tabular}

\section{CONCLUSIONS}

The results presented in this work have confirmed that the new approach to calculate TFR entropy takes advantages compared with the classical spectral power entropy. The new proposed entropy indexes (EnPAins, EnPAsp, EnCOins and $E n C O s p$ ) tend to decrease in high risk group of suffering sudden cardiac death in ischemic cardiomyopathy. Thus, confirming the paradigm that pathology reduces the complexity of the cardiovascular control. Finally, the degree of complexity of this cardiovascular control depends on the physiological state of the subject (daytime versus nighttime period) and on the severity of pathological condition.

\section{ACKNOWLEDGMENT}

CIBER is an initiative of ISCIII. This work was supported within the framework of the CICYT grants TEC2013-44666$\mathrm{R}$ and TEC2014-60337-R from the Spanish Government.

\section{REFERENCES}

[1] M. Heron, D.L. Hoyert, S.L. Murphy, J. Xu, K.D. Kochanek and B. Tejada-Vera, "Deaths: final data for 2006," (available at http://www.cdc.gov/nchs/products/nvsr.htm\#vol57), n. 14, 2009.

[2] Task Force of the European Society of Cardiology and the North American Society of Pacing and Electrophysiology, "Heart rate variability. Standards of measurement, physiological interpretation, and clinical use," Circulation , vol. 93, pp. 1043-1065, 1996.

[3] A. Porta, T. Gnecchi-Ruscone, E. Tobaldini, S. Guzzetti, R. Furlan and N. Montano, "Progressive decrease of heart rate period variability entropy-based complexity during graded head-up tilt," J. Appl. Physiol., vol. 103, pp. 1143-1149, 2007.

[4] A. Voss, R. Schroeder, M. Vallverdú, I. Cygankiewicz, R. Vazquez, A. Bayés de Luna and P. Caminal, "Linear and nonlinear heart rate variability risk stratification in heart failure patients," Comput. Cardiol., vol. 35, pp. 557-560, 2008.

[5] Y-L. Ho, C Lin, Y-H. Lin and M-T. Lo, "The prognostic value of nonlinear analysis of heart rate variability in patients with congestive heart failure - a pilot study of multiscale entropy," PLoS One 6 e18699, 2011.

[6] L. Cohen, "Time-Frequency Analysis", Prentice Hall Signal Processing Series, Prentice Hall: Englewood Cliffs, NJ, USA, 1995.

[7] P. Flandrin, "Time-Frequency and Time-Scale Analysis," Academic: San Diego, CA, USA, 1999.

[8] W.J. Williams, M.L. Brown, A.O. Hero, "Uncertainty, information, and time-frequency distributions," Proc. SPIE Int. Soc. Opt. Eng, vol. 1566, pp. 144-156, 1991.

[9] R.G. Baraniuk, P. Flandrin, A.J.E.M. Janssen, O.J.J. Michel, "Measuring Time-frequency information content using the Rényi entropies," IEEE Trans. Inf. Theory, vol. 47, pp. 1391-1409, 2001.

[10] U. Melia, F. Claria, M. Vallverdu, and P. Caminal, "Measuring Instantaneous and Spectral Information Entropies by Shannon Entropy of Choi-Williams Distribution in the Context of Electroencephalography," Entropy, vol. 16, pp. 2530-2548, 2014.

[11] S.M Pincus, "Approximate entropy as a measure of system complexity," Proc. Natl. Acad. Sci. USA, vol. 88, pp. 2297-2301, 1991.

[12] J. Escudero, R. Hornero, D. Abasolo, "Interpretation of the automutual information rate of decrease in the context of biomedical signal analysis. Application to electroencephalogram recordings," Physiol. Meas, vol. 30, pp. 187-199, 2009.

[13] B. Davies, "Exploring Chaos theory And Experiment," Perseus Books: Reading, MA, USA, 1999.

[14] U. Melia, F. Clariá, M. Vallverdú and P. Caminal, "Filtering and thresholding the analytic signal envelope in order to improve peak and spike noise reduction in EEG signals," Medical engineering \& Physics, vol. 36, pp. 547-553, 2014.

[15] F. Clariá, M. Vallverdú, J. Riba, S. Romero, M.J. Barbanoj, P.Caminal, "Characterization of the cerebral activity by timefrequency representation of evoked EEG potentials," Physiol. Meas., vol. 32, pp. 1327-1346, 2011. 